\title{
FEM SIMULATION OF TRIPLE DIFFUSIVE NATURAL CONVECTION ALONG INCLINED PLATE IN POROUS MEDIUM: PRESCRIBED SURFACE HEAT, SOLUTE AND NANOPARTICLES FLUX
}

\author{
M. GOYAL, R. GOYAL ${ }^{*}$ and R. BHARGAVA \\ Department of Mathematics \\ Indian Institute of Technology Roorkee \\ Roorkee 247667, Uttarakhand, INDIA \\ E-mails: goyalmania87@gmail.co; rangoligoyal@gmail.com; rbharfma@iitr.ernet.in
}

\begin{abstract}
In this paper, triple diffusive natural convection under Darcy flow over an inclined plate embedded in a porous medium saturated with a binary base fluid containing nanoparticles and two salts is studied. The model used for the nanofluid is the one which incorporates the effects of Brownian motion and thermophoresis. In addition, the thermal energy equations include regular diffusion and cross-diffusion terms. The vertical surface has the heat, mass and nanoparticle fluxes each prescribed as a power law function of the distance along the wall. The boundary layer equations are transformed into a set of ordinary differential equations with the help of group theory transformations. A wide range of parameter values are chosen to bring out the effect of buoyancy ratio, regular Lewis number and modified Dufour parameters of both salts and nanofluid parameters with varying angle of inclinations. The effects of parameters on the velocity, temperature, solutal and nanoparticles volume fraction profiles, as well as on the important parameters of heat and mass transfer, i.e., the reduced Nusselt, regular and nanofluid Sherwood numbers, are discussed. Such problems find application in extrusion of metals, polymers and ceramics, production of plastic films, insulation of wires and liquid packaging.
\end{abstract}

Key words: triple diffusive, natural convection, nanofluid, binary base fluid, porous medium, solutal concentration flux, nanoparticle concentration flux, inclined plate.

\section{Introduction}

Research on convective heat transfer in porous media has grown steadily during the past several decades due to its wide applications in civil, mechanical, and chemical engineering. These applications include crude oil extraction, food processing and storage, thermal energy storage, geophysical systems, electro- chemistry, metallurgy, underground disposal of nuclear or non-nuclear waste, cooling system of electronic devices, etc. Excellent reviews of the topics can be found in references by Nield and Bejan [1], Pop and Ingham [2] and Ingham and [3].

Convective heat transfer can be enhanced passively by changing the flow geometry, boundary conditions, or by enhancing thermal conductivity of the fluid. It is well known that conventional heat transfer fluids, including oil, water, and ethylene glycol mixture are poor heat transfer fluids, since the thermal conductivity of these fluids is low. A recent technique to improve the thermal conductivity of these fluids is to add nano-sized metallic particles such as aluminum, titanium, gold, copper, iron or their oxides in the fluid to enhance its thermal properties. The term Nanofluids (nanoparticle fluid suspensions) was coined by Choi and Eastman [4] in 1995, to describe this new class of nanotechnology-based heat transfer fluids that exhibit thermal properties superior to those of their base fluids or conventional particle fluid suspensions. The particles are different from conventional particles (millimeter or microscale) in that they keep suspended in the fluid and no sedimentation occurs. Choi et al. [5] and Masuda et al. [6] showed that even with the small

\footnotetext{
${ }^{*}$ To whom correspondence should be addressed
} 
volumetric fraction of nanoparticles (usually less than 5\%), the thermal conductivity of the base liquid can be enhanced by $10-50 \%$. The enhanced thermal conductivity of a nanofluid together with the thermal dispersion of particles and turbulence induced by their motion contributes to a remarkable improvement in the convective heat transfer coefficient.

A comprehensive survey of convective transport in nanofluids was made by Buongiorno [7]. He proposed a mathematical model to capture the nanoparticle/base fluid slip by treating the nanofluid as a two component mixture with Brownian diffusion and thermophoresis as the important factors in the convective transport process in a nanofluid, which were introduced in the conservation equations for mass and energy.

Research in natural convective heat transfer in nanofluids have found a fast pace in the last few years. Kuznetsov and Nield [8] extended the classical problem of natural convection of a regular fluid over an isothermal vertical plate to the flow with a nanofluid taking into account the combined effects of heat and mass transfer in the presence of Brownian motion and thermophoresis as proposed by Buongiorno [7] and later by Nield and Kuznetsov [9] and Cheng-Minkowycz problem [10]. Khan and Pop [11] studied the steady boundary layer flow of a nanofluid past a stretching sheet and Khan and Aziz [12] investigated the boundary layer flow of a nanofluid past a vertical plate with prescribed heat, solute and nanopartilces flux using the Buongiorno [7] model. Kuznetsov and Nield [13] considered double-diffusive (in reality a triple diffusive) natural convection process from a vertical surface to a binary base fluid containing solute (e.g., salt) as well as nanoparticles. Rana et al. [14] studied the mixed convection boundary layer flow of a nanofluid along an inclined plate embedded in a porous medium. Murthy et al. [15] also investigated the double-diffusive free convection flow over an inclined plate embedded in a non-Darcy porous medium saturated with a nanofluid. In a very recent paper, Khan et al. [16] studied a triple convective-diffusive fluid mixture embedded in a porous medium saturated by a nanofluid on a horizontal plate heated from below and salted from above and below.

It should be, however, mentioned that Nield and Kuznetsov [9, 17] observed that nanoparticles are suspended in the nanofluid using either surfactant or surface charge technology. It is very important to explain how a nanofluid flow is possible in a porous medium. Without special precautions, nanoparticles will be simply absorbed by the porous matrix. Basically, the porous matrix will work as a filter for nanoparticles. This situation has been very well described, explained, and modeled in the recent papers by Wu et al. [18, 19]. The physical situation described in shows that the work on porous media filled by nanofluids are not just a mathematical exercise, but are based on deep physical understanding of nanofluid flows.

Recently, Rionero [20] showed that the presence of more than one chemical dissolved in fluid mixtures is required for describing natural phenomena (contaminant transport, underground water flow, acid rain effects, warning of the stratosphere). The present paper investigates a triple convective-diffusive fluid mixture flow on an inclined plate salted from above and below embedded in a porous medium filled by a water-based nanofluid containing two salts with heat, mass and nanoparticle fluxes. The objective of this study is to investigate cross-diffusion in nanofluids, with the aim of making a detailed comparison with regular cross diffusion effects and the cross-diffusion effects peculiar to nanofluids, and at the same time studying the interaction between these effects when the base fluid of the nanofluid contains two different salts.

\section{Mathematical analysis}

Consider a two-dimensional, incompressible, steady, laminar boundary layer natural convection of a nanofluid past an inclined plate embedded in a porous medium saturated with two different salts having different properties, with an acute angle $\delta$ to the vertical. The $x$-axis is along the plate and the $y$-axis is normal to the plate. The plate is imposed to the surface heat flux, solutal flux of two different salts and the nanoparticle flux, each are prescribed as functions of the distance along the plate $(x)$. The flow in the homogeneous porous medium with the porosity $\varepsilon$ and the permeability $K$ is considered as a Darcy flow, and the Oberbeck-Boussinesq approximation is employed. The porous medium and the nanofluid are assumed in the local thermal equilibrium. In addition, the thermal energy equations include regular diffusion and crossdiffusion terms for components of salts having concentration, $C_{1}$ and $C_{2}$. Following Rionero [20], we assume that two different chemical components ("salts") $S_{m}(m=1,2)$ are dissolved in the fluid-saturated porous medium, which have concentrations $C_{m}(m=1,2)$ respectively. At the large distance from the plate $y \rightarrow \infty$ 
the temperature, solutal concentrations of both salts and the nanoparticle concentration are represented by $T_{\infty}, C_{1 \infty}, C_{2 \infty}$ and $\phi_{\infty}$, respectively. Defining the stream function $\psi$ such that

$$
u=\frac{\partial \psi}{\partial y}, \quad v=-\frac{\partial \psi}{\partial x}
$$

the continuity equation is automatically satisfied. The five field equations representing the conservation of momentum, thermal energy, both solutes and nanoparticles for nanofluids over a heated surface embedded in a saturated porous medium, can be written as

$$
\begin{aligned}
& \frac{\partial^{2} \psi}{\partial y^{2}}=\frac{\left(1-\phi_{\infty}\right) \rho f_{\infty} g K \cos (\delta)}{\mu}\left(\beta_{T} \frac{\partial T}{\partial y}+\beta_{C_{1}} \frac{\partial C_{1}}{\partial y}+\beta_{C_{2}} \frac{\partial C_{2}}{\partial y}\right)-\frac{\left(\rho_{p}-\rho f_{\infty}\right) g K \cos (\delta)}{\mu} \frac{\partial \phi}{\partial y} \\
& \frac{\partial \psi}{\partial y} \frac{\partial T}{\partial x}-\frac{\partial \psi}{\partial x} \frac{\partial T}{\partial y}=\alpha_{m} \frac{\partial^{2} T}{\partial y^{2}}+\tau\left(D_{B} \frac{\partial \phi}{\partial y} \frac{\partial T}{\partial y}+\frac{D_{T}}{T_{\infty}}\left(\frac{\partial T}{\partial y}\right)^{2}\right)+\sigma\left(D_{T C_{1}} \frac{\partial^{2} C_{1}}{\partial y^{2}}+D_{T C_{2}} \frac{\partial^{2} C_{2}}{\partial y^{2}}\right) \\
& \frac{\partial \psi}{\partial y} \frac{\partial C_{1}}{\partial x}-\frac{\partial \psi}{\partial x} \frac{\partial C_{1}}{\partial y}=\epsilon D_{S 1} \frac{\partial^{2} C_{1}}{\partial y^{2}}+\epsilon D_{C I T} \frac{\partial^{2} T}{\partial y^{2}} \\
& \frac{\partial \psi}{\partial y} \frac{\partial C_{2}}{\partial x}-\frac{\partial \psi}{\partial x} \frac{\partial C_{2}}{\partial y}=\epsilon D_{S 2} \frac{\partial^{2} C_{2}}{\partial y^{2}}+\epsilon D_{C 2 T} \frac{\partial^{2} T}{\partial y^{2}} \\
& \frac{\partial \psi}{\partial y} \frac{\partial \phi}{\partial x}-\frac{\partial \psi}{\partial x} \frac{\partial \phi}{\partial y}=\epsilon D_{B} \frac{\partial^{2} \phi}{\partial y^{2}}+\epsilon \frac{D_{T}}{T_{\infty}} \frac{\partial^{2} T}{\partial y^{2}}
\end{aligned}
$$

where, the boundary conditions are given as follows

$$
\begin{aligned}
& v=0, \quad-k \frac{d T}{d y}=q_{w}(x), \quad-D_{S 1} \frac{\partial C_{1}}{\partial y}=q_{m l}(x), \\
& -D_{S 2} \frac{\partial C_{2}}{\partial y}=q_{m 2}(x), \quad-D_{B} \frac{\partial \phi}{\partial y}=q_{n p}(x) \quad \text { at } \quad y=0, \\
& u=v=0, \quad T \rightarrow T_{\infty}, \quad C_{1} \rightarrow C_{1 \infty}, \quad C_{2} \rightarrow C_{2 \infty}, \quad \phi \rightarrow \phi_{\infty} \quad \text { as } \quad y \rightarrow \infty .
\end{aligned}
$$

Using dimensionless variables as

$$
\begin{aligned}
& \eta=\frac{y}{x} R a_{x}^{1 / 3}, \\
& f(\eta)=\frac{\psi}{\alpha_{m} R a_{x}^{\frac{1}{3}}}, \quad \theta(\eta)=\frac{T-T_{\infty}}{\left(q_{w} x / k\right)} R a_{x}^{\frac{1}{3}}, \quad \gamma_{1}(\eta)=\frac{C_{1}-C_{l \infty}}{\left(q_{m 1} x / D_{S 1}\right)} R a_{x}^{\frac{1}{3}}, \\
& \gamma_{2}(\eta)=\frac{C_{2}-C_{2 \infty}}{\left(q_{m 2} x / D_{S 2}\right)} R a_{x}^{1 / 3}, \quad \varphi(\eta)=\frac{\phi-\phi_{\infty}}{\left(q_{n p} x / D_{B}\right)} R a_{x}^{1 / 3},
\end{aligned}
$$


with the local Rayleigh number defined as

$$
R a_{x}=\frac{(1-\phi) \rho f_{\infty} \beta g x q_{w} x}{\mu \alpha_{m} k} .
$$

Using the similarity transformation Eqs (2.10), the governing Eqs (2.2)-(2.6) reduce the following four coupled, nonlinear ordinary differential equations

$$
\begin{aligned}
& f^{\prime \prime}-\left(\theta^{\prime}+N_{C 1} \gamma_{1}^{\prime}+N_{C 2} \gamma_{2}^{\prime}-N r \varphi^{\prime}\right) \cos (\delta)=0, \\
& \theta^{\prime \prime}-\frac{2 \lambda+1}{3} f^{\prime} \theta+\frac{\lambda+2}{3} f \theta^{\prime}+N b \theta^{\prime} \varphi^{\prime}+N t \theta^{\prime 2}+N d_{1} \gamma_{1}^{\prime \prime}+N d_{2} \gamma_{2}^{\prime \prime}=0, \\
& \gamma_{1}^{\prime \prime}-\frac{2 \lambda+1}{3} L e_{1} f^{\prime} \gamma_{1}+\frac{\lambda+2}{3} L e_{1} f \gamma_{1}^{\prime}+L d_{1} \theta^{\prime \prime}=0, \\
& \gamma_{2}^{\prime \prime}-\frac{2 \lambda+1}{3} L e_{2} f^{\prime} \gamma_{2}+\frac{\lambda+2}{3} L e_{2} f \gamma_{2}^{\prime}+L d_{2} \theta^{\prime \prime}=0, \\
& \varphi^{\prime \prime}-\frac{2 \lambda+1}{3} L n f^{\prime} \varphi+\frac{\lambda+2}{3} \operatorname{Lnf} \varphi^{\prime}+\frac{N t}{N b} \theta^{\prime \prime}=0 .
\end{aligned}
$$

The transformed boundary conditions are

$$
\begin{aligned}
& f(0)=0, \quad f^{\prime}(0)=1, \quad \theta^{\prime}(0)=-1, \quad \gamma_{1}^{\prime}(0)=-1, \quad \gamma_{2}^{\prime}(0)=-1, \quad \varphi^{\prime}(0)=-1 \text { at } \eta=0, \\
& f^{\prime}(\eta) \rightarrow 0, \quad \theta(\eta) \rightarrow 0, \quad \gamma_{1}(\eta) \rightarrow 0, \quad \gamma_{2}(\eta) \rightarrow 0, \quad \varphi(\eta) \rightarrow 0 \text { as } \eta \rightarrow \infty
\end{aligned}
$$

where prime denotes the differentiation with respect to ' $\eta$ '. The various parameters are defined as

$$
\begin{aligned}
& N c_{1}=\frac{\beta_{C 1}\left[q_{m 1}(x) / D_{S 1}\right]}{\beta_{T}\left[q_{w}(x) / k\right]}, \quad N c_{2}=\frac{\beta_{C 2}\left[q_{m 2}(x) / D_{S 2}\right]}{\beta_{T}\left[q_{w}(x) / k\right]}, \quad N r=\frac{\left(\rho_{p}-\rho_{f \infty}\right)\left[q_{n p}(x) / D_{B}\right]}{\rho_{\infty}\left(1-\phi_{\infty}\right) \beta_{T}\left[q_{w}(x) / k\right]}, \\
& N b=\frac{\tau D_{B}\left[x q_{n p}(x) / D_{B}\right]}{\alpha_{m} R a_{x}^{1 / 3}}, N t=\frac{\tau D_{T}\left[x q_{w}(x) / k\right]}{\alpha_{m} T_{\infty} R a_{x}^{1 / 3}}, \quad N d_{1}=\frac{\sigma D_{T C 1}\left[q_{m 1}(x) / D_{S 1}\right]}{\alpha_{m}\left[q_{w}(x) / k\right]}, \\
& N d_{2}=\frac{\sigma D_{T C 2}\left[q_{m 2}(x) / D_{S 2}\right]}{\alpha_{m}\left[q_{w}(x) / k\right]}, \quad L e_{1}=\frac{\alpha_{m}}{\epsilon D_{S 1}}, L e_{2}=\frac{\alpha_{m}}{\epsilon D_{S 2}}, \quad L n=\frac{\alpha_{m}}{\epsilon D_{B}}, \\
& L d_{1}=\frac{D_{C I T}\left[q_{w}(x) / k\right]}{D_{S 1}\left[q_{m 1}(x) / D_{S 1}\right]}, \quad L d_{2}=\frac{D_{C 2 T}\left[q_{w}(x) / k\right]}{D_{S 2}\left[q_{m 2}(x) / D_{S 2}\right]},
\end{aligned}
$$

with

$$
q_{w}(x)=A_{w} x^{\lambda}, \quad q_{m 1}(x)=A_{m 1} x^{\lambda}, \quad q_{m 2}(x)=A_{m 2} x^{\lambda}, \quad q_{n p}(x)=A_{n p} x^{\lambda} .
$$


The quantities of physical interest are the local Nusselt number, the Sherwood number corresponding to salts $S_{1}$ and $S_{2}$ and the nanofluid Sherwood number, which are defined by

$$
\begin{aligned}
& \mathrm{Nu}_{x}=\frac{x q_{w}(x)}{k\left(T_{w}-T_{\infty}\right)}, \quad \mathrm{Sh}_{x}^{1}=\frac{x q_{m 1}(x)}{D_{S 1}\left(C_{1 w}-C_{1 \infty}\right)}, \quad \mathrm{Sh}_{x}^{2}=\frac{x q_{m 2}(x)}{D_{S 2}\left(C_{2 w}-C_{2 \infty}\right)}, \\
& \mathrm{Sh}_{x, n}=\frac{x q_{n p}(x)}{D_{B}\left(\phi_{w}-\phi_{\infty}\right)} .
\end{aligned}
$$

In terms of dimensionless variables, these local numbers are written as a modified Nusselt number, modified Sherwood numbers and the modified nanofluid Sherwood number, respectively and can be written as

$$
\begin{aligned}
& \operatorname{Ra}_{x}^{\frac{1}{3}} \mathrm{Nu}_{x}=\frac{1}{\theta(0)}=\mathrm{Nu} r, \quad \mathrm{Ra}_{x}^{\frac{1}{3}} \operatorname{Sh}_{x}^{1}=\frac{1}{\gamma_{1}(0)}=\operatorname{Sh} r^{1}, \\
& \operatorname{Ra}_{x}^{\frac{1}{3}} \operatorname{Sh}_{x}^{2}=\frac{1}{\gamma_{2}(0)}=\operatorname{Sh} r^{2}, \quad \operatorname{Ra}_{x}^{1 / 3} \operatorname{Sh}_{x, n}=\frac{1}{\varphi(0)}=\operatorname{Sh} r n .
\end{aligned}
$$

\section{Numerical implementation}

\subsection{Finite Element Method}

The Finite Element Method (FEM) is a computer-based and numerical scheme which is used for solving a different kind of practical engineering problems that occur in many fields, such as heat transfer, fluid mechanics [21], chemical processing [22], rigid body dynamics [23], solid mechanics [24], and many other fields. The system of ODE given by Eqs (2.12)-(2.16) and (2.17), (2.18) is solved using the FEM, which is a robust technique for numerical simulation. Assuming

$$
f^{\prime}=h,
$$

the system of Eqs (2.12)-(2.16) reduces to

$$
\begin{aligned}
& h^{\prime}-\left(\theta^{\prime}+N_{C 1} \gamma_{1}^{\prime}+N_{C 2} \gamma_{2}^{\prime}-N r \varphi^{\prime}\right) \cos (\delta)=0, \\
& \theta^{\prime \prime}-\frac{2 \lambda+1}{3} h \theta+\frac{\lambda+2}{3} f \theta^{\prime}+N b \theta^{\prime} \varphi^{\prime}+N t \theta^{\prime 2}+N d_{1} \gamma_{1}^{\prime \prime}+N d_{2} \gamma_{2}^{\prime \prime}=0, \\
& \gamma_{1}^{\prime \prime}-\frac{2 \lambda+1}{3} L e_{1} h \gamma_{1}+\frac{\lambda+2}{3} L e_{1} f \gamma_{1}^{\prime}+L d_{1} \theta^{\prime \prime}=0, \\
& \gamma_{2}^{\prime \prime}-\frac{2 \lambda+1}{3} L e_{2} h \gamma_{2}+\frac{\lambda+2}{3} L e_{2} f \gamma_{2}^{\prime}+L d_{2} \theta^{\prime \prime}=0, \\
& \varphi^{\prime \prime}-\frac{2 \lambda+1}{3} \operatorname{Lnh} \varphi+\frac{\lambda+2}{3} \operatorname{Lnf} \varphi^{\prime}+\frac{N t}{N b} \theta^{\prime \prime}=0
\end{aligned}
$$

and the corresponding boundary conditions are 


$$
\begin{aligned}
& f(0)=0, \quad h(0)=1, \quad \theta^{\prime}(0)=-1, \quad \gamma_{1}^{\prime}(0)=-1, \quad \gamma_{2}^{\prime}(0)=-1, \quad \varphi^{\prime}(0)=-1 \text { at } \eta=0, \\
& h=0, \quad \theta(\eta) \rightarrow 0, \quad \gamma_{1}(\eta) \rightarrow 0, \quad \gamma_{2}(\eta) \rightarrow 0, \quad \varphi(\eta) \rightarrow 0 \quad \text { as } \quad \eta \rightarrow \infty .
\end{aligned}
$$

The weighted residual formulation associated with Eqs (3.1)-(3.6) over a typical quadratic element is given by

$$
\begin{aligned}
& \int_{\eta_{e}}^{\eta_{e+1}} W_{1}\left\{f^{\prime}-h\right\} d \eta=0, \\
& \int_{\eta_{e}}^{\eta_{e+1}} W_{2}\left\{h^{\prime}-\left(\theta^{\prime}+N_{C 1} \gamma_{1}^{\prime}+N_{C 2} \gamma_{2}^{\prime}-N r \varphi^{\prime}\right) \cos (\delta)\right\} d \eta=0 \\
& \int_{\eta_{e}}^{\eta_{e+1}} W_{3}\left\{\theta^{\prime \prime}-\frac{2 \lambda+1}{3} h \theta+\frac{\lambda+2}{3} f \theta^{\prime}+N b \theta^{\prime} \varphi^{\prime}+N t \theta^{\prime 2}+N d_{1} \gamma_{1}^{\prime \prime}+N d_{2} \gamma_{2}^{\prime \prime}\right\} d \eta=0, \\
& \int_{\eta_{e}}^{\eta_{e+1}} W_{4}\left\{\gamma_{1}^{\prime \prime}-\frac{2 \lambda+1}{3} L e_{1} h \gamma_{1}+\frac{\lambda+2}{3} L e_{1} f \gamma_{1}^{\prime}+L d_{1} \theta^{\prime \prime}\right\} d \eta=0 \\
& \int_{\eta_{e}}^{\eta_{e+1}} W_{5}\left\{\gamma_{2}^{\prime \prime}-\frac{2 \lambda+1}{3} L e_{2} h \gamma_{2}+\frac{\lambda+2}{3} L e_{2} f \gamma_{2}^{\prime}+L d_{2} \theta^{\prime \prime}\right\} d \eta=0, \\
& \int_{\eta_{e}}^{\eta_{e+1}} W_{6}\left\{\varphi^{\prime \prime}-\frac{2 \lambda+1}{3} L n h \varphi+\frac{\lambda+2}{3} L n f \varphi^{\prime}+\frac{N t}{N b} \theta^{\prime \prime}\right\} d \eta=0
\end{aligned}
$$

where $W_{1}, W_{2}, W_{3}, W_{4}, W_{5}$ and $W_{6}$ are arbitrary test functions. Assuming that a typical dependent variable is of the form

$$
\Omega=\sum_{i=1}^{3} \Omega_{i} \psi_{i},
$$

$\psi_{i}$ is defined as the quadratic shape functions

$$
\begin{aligned}
& \psi_{1}^{e}=\frac{\left(\eta_{e+1}-\eta_{e}-2 \eta\right)\left(\eta_{e+1}-\eta\right)}{\left(\eta_{e}, \eta_{e+1}\right)^{2}}, \\
& \psi_{2}^{e}=\frac{4\left(\eta-\eta_{e}\right)\left(\eta_{e+1}-\eta\right)}{\left(\eta_{e}, \eta_{e+1}\right)^{2}}, \quad \psi_{3}^{e}=-\frac{\left(\eta_{e+1}-\eta_{e}-2 \eta\right)\left(\eta-\eta_{e}\right)}{\left(\eta_{e}, \eta_{e+1}\right)^{2}} .
\end{aligned}
$$

Substituting Eq.(3.16) into the weighted residual formulations Eqs (3.9)-(3.14) of the given differential equations. The finite element model of the equations thus formed is given by 


$$
\left.\begin{array}{llllll}
{\left[K^{11}\right]} & {\left[K^{12}\right]} & {\left[K^{13}\right]} & {\left[K^{14}\right]} & {\left[K^{15}\right]} & {\left[K^{16}\right]} \\
{\left[K^{21}\right]} & {\left[K^{22}\right]} & {\left[K^{23}\right]} & {\left[K^{24}\right]} & {\left[K^{25}\right]} & {\left[K^{26}\right]} \\
{\left[K^{31}\right]} & {\left[K^{32}\right]} & {\left[K^{33}\right]} & {\left[K^{34}\right]} & {\left[K^{35}\right]} & {\left[K^{36}\right]} \\
{\left[K^{41}\right]} & {\left[K^{42}\right]} & {\left[K^{43}\right]} & {\left[K^{44}\right]} & {\left[K^{45}\right]} & {\left[K^{46}\right]} \\
{\left[K^{51}\right]} & {\left[K^{52}\right]} & {\left[K^{53}\right]} & {\left[K^{54}\right]} & {\left[K^{55}\right]} & {\left[K^{56}\right]} \\
{\left[K^{61}\right]} & {\left[K^{62}\right]} & {\left[K^{63}\right]} & {\left[K^{64}\right]} & {\left[K^{65}\right]} & {\left[K^{66}\right]}
\end{array}\right]\left[\begin{array}{l}
\{f\} \\
\{\theta\} \\
\left\{\gamma_{1}\right\} \\
\left\{\gamma_{2}\right\} \\
\{\varphi\}
\end{array}\right]=\left[\begin{array}{l}
\left\{b^{1}\right\} \\
\left\{b^{2}\right\} \\
\left\{b^{3}\right\} \\
\left\{b^{4}\right\} \\
\left\{b^{5}\right\} \\
\left\{b^{6}\right\}
\end{array}\right],
$$

with

$K_{i j}^{11}=\int_{\eta_{e}}^{\eta_{e+1}} \psi_{i} \frac{\partial \psi_{j}}{\partial \eta} d \eta, \quad K_{i j}^{12}=-\int_{\eta_{e}}^{\eta_{e+1}} \psi_{i} \psi_{j} d \eta, \quad K_{i j}^{13}=K_{i j}^{14}=K_{i j}^{15}=K_{i j}^{16}=0$,

$K_{i j}^{21}=0, \quad K_{i j}^{22}=-\int_{\eta_{e}}^{\eta_{e+1}} \psi_{i} \frac{\partial \psi_{j}}{\partial \eta} d \eta, \quad K_{i j}^{23}=K_{i j}^{24}=K_{i j}^{25}=K_{i j}^{26}=-\cos (\delta) \int_{\eta_{e}}^{\eta_{e+1}} \psi_{i} \frac{\partial \psi_{j}}{\partial \eta} d \eta$,

$K_{i j}^{31}=K_{i j}^{32}=K_{i j}^{36}=0$,

$K_{i j}^{33}=\int_{\eta_{e}}^{\eta_{e+1}} \frac{\partial \psi_{i}}{\partial \eta} \frac{\partial \psi_{j}}{\partial \eta} d \eta-\frac{2 \lambda+1}{3} \int_{\eta_{e}}^{\eta_{e+1}} \psi_{i} \bar{h} \psi_{j} d \eta+\frac{\lambda+2}{3} \int_{\eta_{e}}^{\eta_{e+1}} \psi_{i} \bar{f} \frac{\partial \psi_{j}}{\partial \eta} d \eta+$

$+N b \int_{\eta_{e}}^{\eta_{e+1}} \psi_{i} \bar{\varphi}^{\prime} \frac{\partial \psi_{j}}{\partial \eta} d \eta+N t \int_{\eta_{e}}^{\eta_{e+1}} \psi_{i} \bar{\theta}^{\prime} \frac{\partial \psi_{j}}{\partial \eta} d \eta$

$K_{i j}^{34}=-N d_{1} \int_{\eta_{e}}^{\eta_{e+1}} \frac{\partial \psi_{i}}{\partial \eta} \frac{\partial \psi_{j}}{\partial \eta} d \eta, \quad K_{i j}^{35}=-N d_{2} \int_{\eta_{e}}^{\eta_{e+1}} \frac{\partial \psi_{i}}{\partial \eta} \frac{\partial \psi_{j}}{\partial \eta} d \eta$

$K_{i j}^{41}=K_{i j}^{42}=K_{i j}^{45}=K_{i j}^{46}=0, \quad K_{i j}^{43}=-L d_{1} \int_{\eta_{e}}^{\eta_{e+1}} \frac{\partial \psi_{i}}{\partial \eta} \frac{\partial \psi_{j}}{\partial \eta} d \eta$

$K_{i j}^{44}=\int_{\eta_{e}}^{\eta_{e+1}} \frac{\partial \psi_{i}}{\partial \eta} \frac{\partial \psi_{j}}{\partial \eta} d \eta-L e_{1} \frac{2 \lambda+1}{3} \int_{\eta_{e}}^{\eta_{e+1}} \psi_{i} \bar{h} \psi_{j} d \eta+L e_{1} \frac{\lambda+2}{3} \int_{\eta_{e}}^{\eta_{e+1}} \psi_{i} \bar{f} \frac{\partial \psi_{j}}{\partial \eta} d \eta$,

$K_{i j}^{51}=K_{i j}^{52}=K_{i j}^{54}=K_{i j}^{56}=0, \quad K_{i j}^{53}=-L d_{2} \int_{\eta_{e}}^{\eta_{e+1}} \frac{\partial \psi_{i}}{\partial \eta} \frac{\partial \psi_{j}}{\partial \eta} d \eta$,

$K_{i j}^{55}=-\int_{\eta_{e}}^{\eta_{e+1}} \frac{\partial \psi_{i}}{\partial \eta} \frac{\partial \psi_{j}}{\partial \eta} d \eta-L e_{2} \frac{2 \lambda+1}{3} \int_{\eta_{e}}^{\eta_{e+1}} \psi_{i} \bar{h} \psi_{j} d \eta+L e_{2} \frac{\lambda+2}{3} \int_{\eta_{e}}^{\eta_{e+1}} \psi_{i} f^{\prime} \frac{\partial \psi_{j}}{\partial \eta} d \eta$, 


$$
\begin{aligned}
& K_{i j}^{61}=K_{i j}^{62}=K_{i j}^{64}=K_{i j}^{65}=0, \quad K_{i j}^{63}=-\frac{N t}{N b} \int_{\eta_{e}}^{\eta_{e+1}} \frac{\partial \psi_{i}}{\partial \eta} \frac{\partial \psi_{j}}{\partial \eta} d \eta \\
& K_{i j}^{66}=-\int_{\eta_{e}}^{\eta_{e+1}} \frac{\partial \psi_{i}}{\partial \eta} \frac{\partial \psi_{j}}{\partial \eta} d \eta-\operatorname{Ln} \frac{2 \lambda+1}{3} \int_{\eta_{e}}^{\eta_{e+1}} \psi_{i} \bar{h} \psi_{j} d \eta+L n \frac{\lambda+2}{3} \int_{\eta_{e}}^{\eta_{e+1}} \psi_{i} \bar{f} \frac{\partial \psi_{j}}{\partial \eta} d \eta .
\end{aligned}
$$

\section{Validation of the proposed method}

An extensive mesh testing procedure was conducted to ensure a grid-independence solution of a given boundary value problem. The present code was tested for grid independence by calculating the reduced Nusselt, reduced Sherwood and reduced nanofluid Sherwood number on the wall of the sheet. Different combinations of the mesh were explored for $\lambda=0.5, \delta=\frac{\pi}{6}, L d_{1}=1, d_{2}=2, L n=2, L e_{1}=1, L e_{2}=2$, $N d_{1}=0.1, N d_{2}=0.2, N c_{1}=1, N c_{2}=2, N b=0.3, N t=0.2, N r=0.1$, as shown in Tab.1. It has been observed that in the same domain the accuracy is not affected even if the number of elements is increased or the size of the elements is decreased. This serves only to increase compilation times and does not enhance in any way the accuracy of the solutions. Thus, for computational purposes, 6000 elements $\left(h_{e}=0.001\right)$ are taken for presentation of the results. It was verified that the same grid size $(0.001)$ ensures the grid independent solution for all functions. For computational purposes, the region of integration $\eta$ is computed as 0 to $\eta_{\infty}=6$, where $\eta_{\infty}$ corresponds to $\eta \rightarrow \infty$ which lies very well outside the momentum and thermal boundary layers. It has been observed that for the moderate values of $\eta_{\infty}(>6.0)$, there is no appreciable effect on the results. Therefore for computational purposes, infinity has been set as 6.0 .

Table 1. Reduced Nusselt number, Sherwood number of both salts and nanofluid Sherwood number when $\lambda=0.5 ; \delta=\pi / 6 ; L d_{1}=1 ; L d_{2}=2 ; L n=2 ; L e_{1}=1 ; L e_{2}=2 ; N d_{1}=0.1 ; N d_{2}=0.2 ; N c_{1}=1$;

\begin{tabular}{|c|c|c|c|c|c|c|c|}
\hline step size & Nur & & Shr $^{1}$ & & $\mathrm{Shr}^{2}$ & Shrn & \\
\hline & $\eta_{\infty}=4$ & $\eta_{\infty}=6$ & $\eta_{\infty}=8 \quad \eta_{\infty}=4$ & $\eta_{\infty}=6$ & $\eta_{\infty}=8 \quad \eta_{\infty}=4$ & $\eta_{\infty}=6 \quad \eta_{\infty}=8 \eta_{\infty}=4$ & $\eta_{\infty}=6 \quad \eta_{\infty}=8$ \\
\hline 0.2 & 1.109771 & 1.128634 & 1.1546700 .941184 & 0.956687 & 0.9803731 .139247 & 1.1654491 .2030621 .596625 & 1.6488561 .721340 \\
\hline 0.1 & 1.147628 & 1.161983 & 1.1692080 .975086 & 0.987258 & 0.9939931 .192962 & 1.2138001 .2244311 .700656 & 1.7417821 .762074 \\
\hline 0.04 & 1.169524 & 1.172829 & 1.1764140 .995569 & 0.997505 & 1.0008761 .225093 & 1.2297801 .2350551 .761945 & 1.7721331 .782144 \\
\hline 0.02 & 1.176730 & 1.178239 & 1.1788281 .002473 & 1.002729 & 1.0032381 .235726 & 1.2377481 .2386021 .782007 & 1.7870961. \\
\hline 0.01 & 1.180367 & 1.180067 & 1.1808001 .006074 & 1.004550 & 1.0052361 .241060 & 1.2404201 .2414671 .791876 & 1.7920091 .793952 \\
\hline 0.001 & 1.181629 & 1.181007 & 1.1813151 .007401 & 1.005525 & 1.0057901 .242874 & 1.2417741 .2422001 .795077 & 1.7944221 .795217 \\
\hline 0.0001 & 1.182736 & 1.182001 & 1.1819881 .008680 & 1.006626 & 1.0065431 .244401 & 1.2431711 .2431381 .797504 & 1.7967561 .796761 \\
\hline
\end{tabular}
$N c_{2}=2 ; \mathrm{Nb}=0.3 ; \mathrm{Nt}=0.2 ; \mathrm{Nr}=0.1$.

In the present study, the reduced Nusselt number Nur, reduced local Sherwood number of both salts $S h r^{l}, S h r^{2}$ and reduced nanofluid Sherwood number Shrn, defined in Eq.(2.22), are the important characteristics. The numerical values of Nur, Shr ${ }^{1}, S h r^{2}$, Shrn are exhibited in Tabs 1-5. 
Table 2. Comparison of the reduced Nusselt number Nur for free convection along a vertical at plate in a porous medium saturated with nanofluids when $\lambda=-0.5 ; \delta=0 ; L d_{1}=L d_{2}=0 ; N c_{1}=N c_{2}=0 ; N d_{1}$ $=\mathrm{Nd}_{2}=0$.

\begin{tabular}{|c|c|c|c|c|c|c|c|c|c|}
\hline \multirow[t]{3}{*}{$\mathrm{Nb}$} & \multirow[t]{3}{*}{$\mathrm{Nt}$} & \multicolumn{4}{|c|}{$\mathrm{Nr}=0.1$} & \multicolumn{4}{|c|}{$\mathrm{Nr}=0.3$} \\
\hline & & \multicolumn{2}{|c|}{$\mathrm{Ln}=10$} & \multicolumn{2}{|c|}{$\mathrm{Ln}=50$} & \multicolumn{2}{|c|}{$\mathrm{Ln}=10$} & \multicolumn{2}{|c|}{$\mathrm{Ln}=50$} \\
\hline & & $\begin{array}{c}\text { Noghrehabadi } \\
\text { et al. }[25]\end{array}$ & $\begin{array}{l}\text { Present } \\
\text { results }\end{array}$ & $\begin{array}{c}\text { Noghrehabadi } \\
\text { et al. }[25]\end{array}$ & $\begin{array}{l}\text { Present } \\
\text { results }\end{array}$ & $\begin{array}{c}\text { Noghrehabadi } \\
\text { et al. }[25]\end{array}$ & $\begin{array}{l}\text { Present } \\
\text { results }\end{array}$ & $\begin{array}{l}\text { Noghrehabadi } \\
\text { et al. }[25]\end{array}$ & $\begin{array}{l}\text { Present } \\
\text { results }\end{array}$ \\
\hline \multirow{5}{*}{0.1} & 0.1 & 0.53523 & 0.535191 & 0.54514 & 0.545016 & 0.52809 & 0.527827 & 0.54364 & 0.543601 \\
\hline & 0.2 & 0.50476 & 0.504583 & 0.51536 & 0.515175 & 0.49568 & 0.495416 & 0.51345 & 0.513365 \\
\hline & 0.3 & 0.47474 & 0.474701 & 0.48519 & 0.485004 & 0.46451 & 0.464364 & 0.48304 & 0.482819 \\
\hline & 0.4 & 0.44505 & 0.444823 & 0.45454 & 0.454440 & 0.43433 & 0.434210 & 0.45230 & 0.452217 \\
\hline & 0.5 & 0.41555 & 0.415402 & 0.42336 & 0.423113 & 0.40493 & 0.404808 & 0.42115 & 0.411001 \\
\hline \multirow{5}{*}{0.3} & 0.1 & 0.51256 & 0.512424 & 0.53280 & 0.532800 & 0.50792 & 0.507717 & 0.53173 & 0.531676 \\
\hline & 0.2 & 0.48406 & 0.484009 & 0.50365 & 0.503556 & 0.47916 & 0.478949 & 0.50252 & 0.502431 \\
\hline & 0.3 & 0.45568 & 0.455555 & 0.47404 & 0.473888 & 0.45073 & 0.450604 & 0.47290 & 0.472818 \\
\hline & 0.4 & 0.42732 & 0.427238 & 0.44389 & 0.443847 & 0.42251 & 0.422509 & 0.44278 & 0.442701 \\
\hline & 0.5 & 0.39888 & 0.398617 & 0.41315 & 0.413131 & 0.39436 & 0.394115 & 0.41210 & 0.411990 \\
\hline \multirow{5}{*}{0.5} & 0.1 & 0.49087 & 0.490707 & 0.52082 & 0.520717 & 0.48688 & 0.486787 & 0.51983 & 0.519703 \\
\hline & 0.2 & 0.46356 & 0.463504 & 0.49214 & 0.492055 & 0.45962 & 0.459515 & 0.49116 & 0.491005 \\
\hline & 0.3 & 0.43625 & 0.436041 & 0.46297 & 0.462882 & 0.43246 & 0.432212 & 0.46203 & 0.461822 \\
\hline & 0.4 & 0.40885 & 0.408802 & 0.43325 & 0.433113 & 0.40531 & 0.405234 & 0.43236 & 0.432007 \\
\hline & 0.5 & 0.38130 & 0.381254 & 0.40291 & 0.402849 & 0.37807 & 0.377956 & 0.40210 & 0.402073 \\
\hline
\end{tabular}

Table 3. Effects of the nanofluid, buoyancy ratio and the modified Dufour parameters on the dimensionless heat transfer rates for aiding flows when $\lambda=0.5 ; \delta=\pi / 6 ; L d_{1}=L d_{2}=0.2 ; L n=2 ; L e_{1}=L e_{2}=$ $1.0 ; \mathrm{Nd}_{2}=0.2 ; \mathrm{Nc}_{2}=5$.

\begin{tabular}{|c|c|c|c|c|c|c|c|c|}
\hline \multirow[t]{2}{*}{$\mathrm{Nr}$} & \multirow[t]{2}{*}{$\mathrm{Nb}$} & \multirow[t]{2}{*}{$\mathrm{Nt}$} & \multicolumn{2}{|c|}{$\mathrm{Nd}_{1}=0.2$} & \multicolumn{2}{|c|}{$\mathrm{Nd}_{1}=0.4$} & \multicolumn{2}{|c|}{$\mathrm{Nd}_{1}=0.6$} \\
\hline & & & $\mathrm{Nc}_{1}=3$ & $\mathrm{Nc}_{1}=5$ & $\mathrm{Nc}_{1}=3$ & $\mathrm{Nc}_{1}=5$ & $\mathrm{Nc}_{1}=3$ & $\mathrm{Nc}_{1}=5$ \\
\hline \multirow[t]{9}{*}{0.1} & 0.1 & 0.1 & 1.456337 & 1.558528 & 1.336233 & 1.428332 & 1.235013 & 1.318645 \\
\hline & & 0.2 & 1.424249 & 1.526410 & 1.307561 & 1.399620 & 1.209034 & 1.292611 \\
\hline & & 0.3 & 1.392251 & 1.494303 & 1.278982 & 1.370927 & 1.183124 & 1.266578 \\
\hline & 0.2 & 0.1 & 1.436034 & 1.537985 & 1.318800 & 1.410684 & 1.219821 & 1.303256 \\
\hline & & 0.2 & 1.406351 & 1.507931 & 1.292192 & 1.383749 & 1.195617 & 1.278766 \\
\hline & & 0.3 & 1.375709 & 1.477037 & 1.264796 & 1.356114 & 1.170761 & 1.253676 \\
\hline & 0.3 & 0.1 & 1.416099 & 1.517772 & 1.301620 & 1.393278 & 1.204786 & 1.288022 \\
\hline & & 0.2 & 1.387014 & 1.488280 & 1.275512 & 1.366797 & 1.181021 & 1.263908 \\
\hline & & 0.3 & 1.356961 & 1.457910 & 1.248608 & 1.339598 & 1.156585 & 1.239189 \\
\hline \multirow[t]{9}{*}{0.2} & 0.1 & 0.1 & 1.45 & 1.55 & 1.332122 & 1.42 & 1288 & 1.315367 \\
\hline & & 0.2 & 1.417543 & 1.520529 & 1.301530 & 1.394318 & 1.203567 & 1.287793 \\
\hline & & 0.3 & 1.383432 & 1.486570 & 1.271044 & 1.363941 & 1.175918 & 1.260219 \\
\hline & 0.2 & 0.1 & 1.432568 & 1.534947 & 1.315694 & 1.407957 & 1.217013 & 1.300786 \\
\hline & & 0.2 & 1.401756 & 1.503925 & 1.288095 & 1.380154 & 1.191917 & 1.275500 \\
\hline & & 0.3 & 1.370112 & 1.472130 & 1.259775 & 1.351699 & 1.166232 & 1.249661 \\
\hline & 0.3 & 0.1 & 1.412973 & 1.515043 & 1.298827 & 1.390821 & 1.202266 & 1.285802 \\
\hline & & 0.2 & 1.383158 & 1.484891 & 1.272066 & 1.363762 & 1.177914 & 1.261165 \\
\hline & & 0.3 & 1.352437 & 1.453928 & 1.244557 & 1.336024 & 1.152927 & 1.235954 \\
\hline
\end{tabular}


Table 4. Effects of Lewis numbers on the dimensionless mass transfer rates of both salts and nanoparticles for aiding flows when $\lambda=0.5 ; \delta=\pi / 6 ; L d_{2}=0.2 ; N c_{1}=N c_{2}=3 ; N d_{1}=0.4 ; N d_{2}=0.2 ; N b=0.3 ; N t=0.2$; $N r=0.1$.

\begin{tabular}{|c|c|c|c|c|c|c|c|c|}
\hline \multirow[t]{2}{*}{$\mathrm{Le}_{1}$} & \multirow[t]{2}{*}{$\mathrm{Le}_{2}$} & \multirow[t]{2}{*}{$\mathrm{Ln}$} & \multicolumn{3}{|c|}{$\mathrm{Ld}_{1}=0.2$} & \multicolumn{3}{|c|}{$\mathrm{Ld}_{1}=0.4$} \\
\hline & & & $\mathrm{Shr}^{1}$ & $\mathrm{Shr}^{2}$ & Shrn & $\mathrm{Shr}^{1}$ & $\mathrm{Shr}^{2}$ & Shrn \\
\hline \multirow[t]{9}{*}{1} & 1 & 1 & 1.571477 & 1.571477 & 1.282409 & 1.460606 & 1.600526 & 1.308128 \\
\hline & & 3 & 1.574553 & 1.574553 & 2.644511 & 1.462636 & 1.603747 & 2.684946 \\
\hline & & 5 & 1.574579 & 1.574579 & 3.619065 & 1.462345 & 1.603789 & 3.669251 \\
\hline & 3 & 1 & 1.428570 & 2.816647 & 1.165602 & 1.338507 & 2.869389 & 1.200049 \\
\hline & & 3 & 1.432154 & 2.823437 & 2.442142 & 1.341060 & 2.876341 & 2.494790 \\
\hline & & 5 & 1.432058 & 2.823791 & 3.361329 & 1.340674 & 2.876839 & 3.426053 \\
\hline & 5 & 1 & 1.394974 & 3.693484 & 1.141650 & 1.311425 & 3.759370 & 1.177920 \\
\hline & & 3 & 1.398634 & 3.702581 & 2.394352 & 1.314050 & 3.768698 & 2.450158 \\
\hline & & 5 & 1.398482 & 3.703231 & 3.297930 & 1.313609 & 3.769586 & 3.366647 \\
\hline \multirow[t]{9}{*}{3} & 1 & 1 & 2.827505 & 1.432798 & 1.173352 & 2.676978 & 1.445940 & 1.185597 \\
\hline & & 3 & 2.834218 & 1.436302 & 2.464986 & 2.682721 & 1.449460 & 2.484634 \\
\hline & & 5 & 2.834508 & 1.436169 & 3.394514 & 2.682421 & 1.449343 & 3.419066 \\
\hline & 3 & 1 & 2.517420 & 2.517419 & 1.007277 & 2.388144 & 2.544280 & 1.026994 \\
\hline & & 3 & 2.525435 & 2.525435 & 2.183759 & 2.395411 & 2.552300 & 2.213883 \\
\hline & & 5 & 2.525344 & 2.525344 & 3.040493 & 2.394801 & 2.552339 & 3.077182 \\
\hline & 5 & 1 & 2.430632 & 3.299892 & 0.971774 & 2.312121 & 3.333055 & 0.993362 \\
\hline & & 3 & 2.438764 & 3.310502 & 2.114235 & 2.319506 & 3.343691 & 2.147411 \\
\hline & & 5 & 2.438444 & 3.310537 & 2.949205 & 2.318673 & 3.343929 & 2.989594 \\
\hline
\end{tabular}

Table 5. Effects of $\mathrm{Nt}$, and $\mathrm{Ln}$ on the dimensionless heat, solutal mass of salts $1 \& 2$ and nanoparticle mass transfer rates for aiding flows when $\lambda=0.5 ; L d_{1}=1 ; L d_{2}=2 ; L e_{1}=1 ; L e_{2}=2 ; N d_{1}=0.1 ; N d_{2}=$ $0.2 ; N c_{1}=1 ; N c_{2}=5 ; N b=0.3 ; N r=0.1$.

\begin{tabular}{|c|c|c|c|c|c|c|c|c|c|}
\hline \multirow[t]{2}{*}{$\mathrm{t}$} & \multirow[t]{2}{*}{$\delta$} & \multicolumn{2}{|c|}{ Nur } & \multicolumn{2}{|c|}{$\mathrm{Shr}^{1}$} & \multicolumn{2}{|c|}{$\mathrm{Shr}^{2}$} & \multicolumn{2}{|c|}{ Shrn } \\
\hline & & $\mathrm{Ln}=5$ & $\mathrm{Ln}=15$ & $\operatorname{Ln}=5$ & $\mathrm{Ln}=15$ & $\mathrm{Ln}=5$ & $\mathrm{Ln}=15$ & $\mathrm{Ln}=5$ & $\mathrm{Ln}=15$ \\
\hline \multirow[t]{4}{*}{0.1} & 0 & 1.296269 & 1.316955 & 1.039764 & 1.036154 & 1.275138 & 1.268796 & 3.567438 & 6.486363 \\
\hline & $\pi / 6$ & 1.231761 & 1.252439 & 0.992472 & 0.989245 & 1.218271 & 1.211820 & 3.405421 & 6.196226 \\
\hline & $\pi / 3$ & 1.145822 & 1.166370 & 0.929671 & 0.926354 & 1.142514 & 1.135902 & 3.189316 & 5.809114 \\
\hline & $\pi / 2$ & 1.011557 & 1.031879 & 0.831695 & 0.828202 & 1.024290 & 1.017391 & 2.850863 & 5.200802 \\
\hline \multirow[t]{4}{*}{0.3} & 0 & 1.229170 & 1.251327 & 1.054892 & 1.051274 & 1.304875 & 1.298065 & 3.045969 & 5.857219 \\
\hline & $\pi / 6$ & 1.164621 & 1.186721 & 1.007865 & 1.004488 & 1.248447 & 1.241550 & 2.912429 & 5.603363 \\
\hline & $\pi / 3$ & 1.078642 & 1.100440 & 0.945392 & 0.941875 & 1.173382 & 1.166248 & 2.734505 & 5.264864 \\
\hline & $\pi / 2$ & 0.944328 & 0.965588 & 0.848037 & 0.844273 & 1.056473 & 1.048919 & 2.456405 & 4.733886 \\
\hline \multirow[t]{4}{*}{0.5} & 0 & 1.161883 & 1.184412 & 1.071913 & 1.068378 & 1.338292 & 1.331253 & 2.711381 & 5.442550 \\
\hline & $\pi / 6$ & 1.097317 & 1.119716 & 1.025254 & 1.021855 & 1.282549 & 1.275464 & 2.598992 & 5.219003 \\
\hline & $\pi / 3$ & 1.011359 & 1.033183 & 0.963302 & 0.959721 & 1.208557 & 1.201187 & 2.449586 & 4.921312 \\
\hline & $\pi / 2$ & 0.877130 & 0.897932 & 0.866924 & 0.863054 & 1.093695 & 1.085844 & 2.217014 & 4.455858 \\
\hline
\end{tabular}

The influence of $N b, N t, N r$ and $L n$ on the reduced Nusselt number is shown in Tab.2. Further, the results are compared with those reported by Noghrehabadi et al. [25]. The comparisons in all the cases are found to be in good agreement.

\section{Results and discussions}

Numerical computations have been carried out for different values of the parameters involved, namely the Prandtl number $(\mathrm{Pr})$, regular Lewis number $\left(\mathrm{Le}_{1}, \mathrm{Le}_{2}\right)$, modified Dufour parameters of both salts $\left(\mathrm{Nd}_{1}, \mathrm{Nd}_{2}\right)$, buoyancy ratio of both salts $\left(N c_{1}, N c_{2}\right)$, Dufour-solutal Lewis number of salts 1 and $2\left(\operatorname{Ld}_{1}, \mathrm{Ld}_{2}\right)$, 
angle of inclination, nanofluid buoyancy ratio $(N r)$, nanofluid Lewis number (Ln), the Brownian motion parameter $(\mathrm{Nb})$ and thermophoresis parameter $(N t)$. The effects of the flow controlling parameters on the dimensionless velocity, temperature, solutal concentrations of both salts, nanoparticle concentration, the rate of heat and mass transfer are investigated and presented graphically in Figs 1-10.

The numerical values of the reduced Nusselt number Nur, reduced local Sherwood number of both salts $\mathrm{Shr}^{1}, \mathrm{Shr}^{2}$ and reduced nanofluid Sherwood number Shrn are given in Tabs 3-5 for various values of parameters. Table 3 depicts the effect of nanofluid buoyancy ratio, nanofluid, and the modified Dufour parameters on the dimensionless heat transfer rates for assisting flow. The results in this table indicate that the increase in the Brownian motion parameter, the thermophoresis parameter, or the buoyancy ratio parameter decreases the heat transfer rates. This table shows that an increase in the modified Dufour parameter increases the heat transfer rates. The variations of the heat transfer in Tab.3 reveal that the effects of the nanofluid parameters on the heat transfer rate are more than that of the nanofluid buoyancy ratio parameter. The effects of the regular and nanofluid Lewis number on the dimensionless mass transfer of both salts and nanoparticles rates are shown in Tab.4. It can be noticed that mass transfer rates of salts $1 \& 2$ and nanoparticles depend upon their respective Lewis numbers.

The variation of the reduced Nusselt number, Sherwood number of both salts and nanofluid Sherwood number with the thermophoretic parameter $N t$, inclination angle $\delta$ and nanofluid Lewis number $\mathrm{Ln}$ is presented in Tab.5 for aiding flows only. Clearly, the heat transfer rate and mass transfer rate of both salts and nanoparticles decreases with an increase in the angle of inclination $\delta$. The heat and mass transfer rate is larger for the case of vertical surfaces $(\delta=0)$ as compared to the horizontal surfaces $(\delta=\pi / 2)$.

Figure 1 shows the profiles of stream function, temperature, solutal concentration of salt $1 \& 2$ and nanoparticle concentration for selected parameters. Figure 2 depicts the effects of all the three types of diffusion on the reduced Nusselt number (dimensionless heat transfer rates) and a comparison of diffusion is also shown in this figure. It can be seen that in the case of a regular fluid (without nanofluids and salts), the dimensionless heat transfer rate is smaller than other diffusions. After the inclusion of nanofluids and salts, the rate of heat transfer increases due to an increase in the thermal conductivity and rate of diffusion of both salts.

Figures $3 \mathrm{a}$ and $3 \mathrm{~b}$ depict the effects of the buoyancy ratio of salt 1 and exponent $\lambda$. The effect of changing the exponent $\lambda$ from 0 to 1.0 is to decrease the local (at a given value of $\eta$ ) values of the surface temperature in both assisting and opposing flows. The buoyancy ratio of salt 1 reduces the dimensionless temperature and hence the thickness of the thermal boundary also reduces in the case of aiding flows, whereas in the case of opposing flows, it helps in increasing the surface temperature.

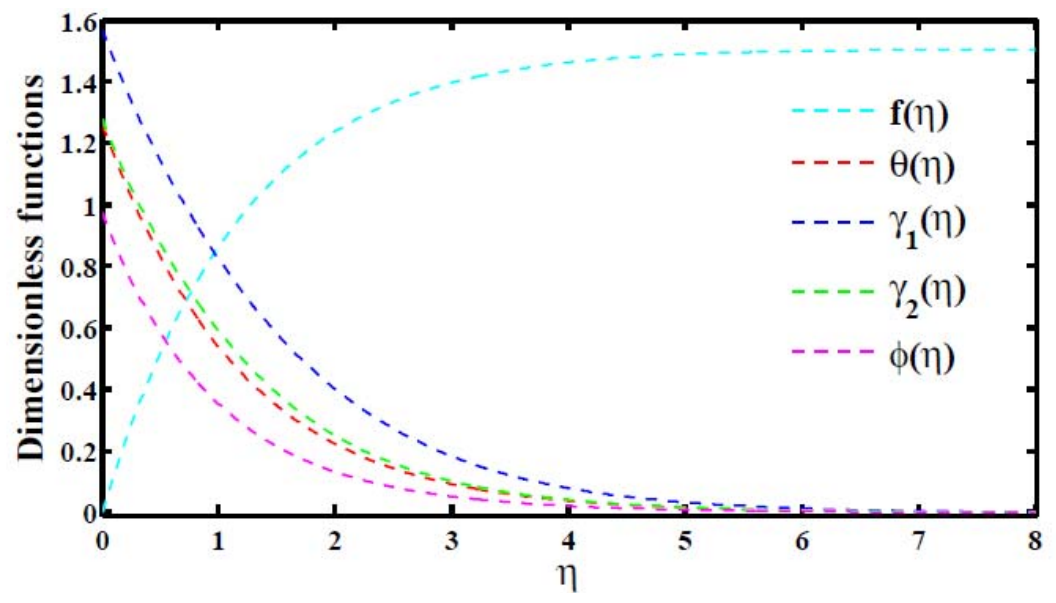

Fig.1. Profile of all dimensionless functions when $\mathrm{Ld}_{1}=1 ; \mathrm{Ld}_{2}=2 ; \mathrm{Le}_{1}=1 ; \mathrm{Le}_{2}=2 ; \mathrm{Ln}=2 ; \mathrm{Nb}=\mathrm{Nt}=0.1$; $\lambda=0.5 ; \delta=\pi / 6 ; \mathrm{Nd}_{1}=0.1 ; \mathrm{Nd}_{2}=0.2 ; \mathrm{Nc}_{1}=\mathrm{Nc}_{2}=\mathrm{Nr}=0.1$. 


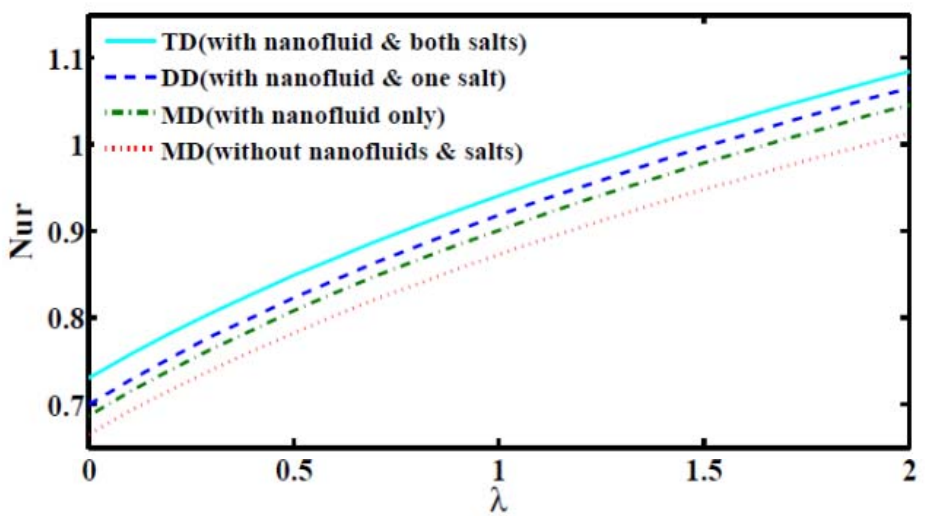

Fig.2. Effects of heat and mass diffusion on the dimensionless heat transfer rate, where TD stands for triple diffusion (nanofluid with both salts), DD stands for double diffusion(nanofluid with salt 1), MD stands for mono diffusion(regular or nanofluids).

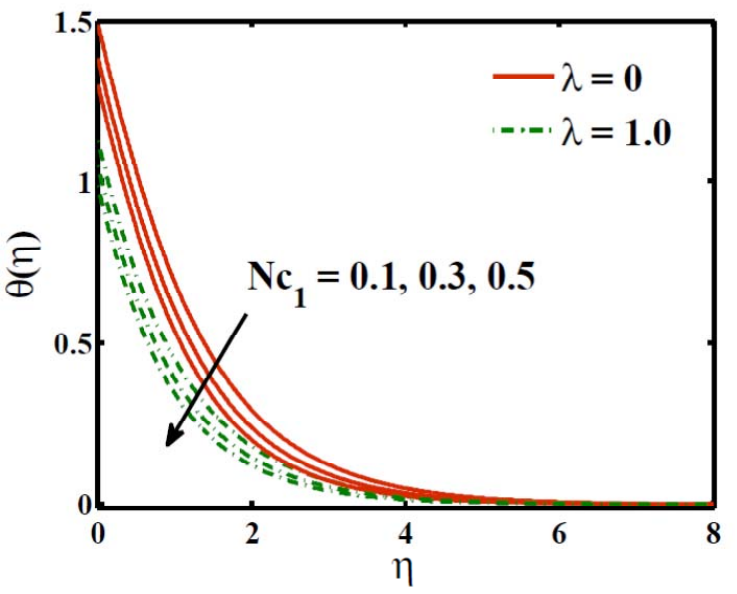

(a) Assisting Flows

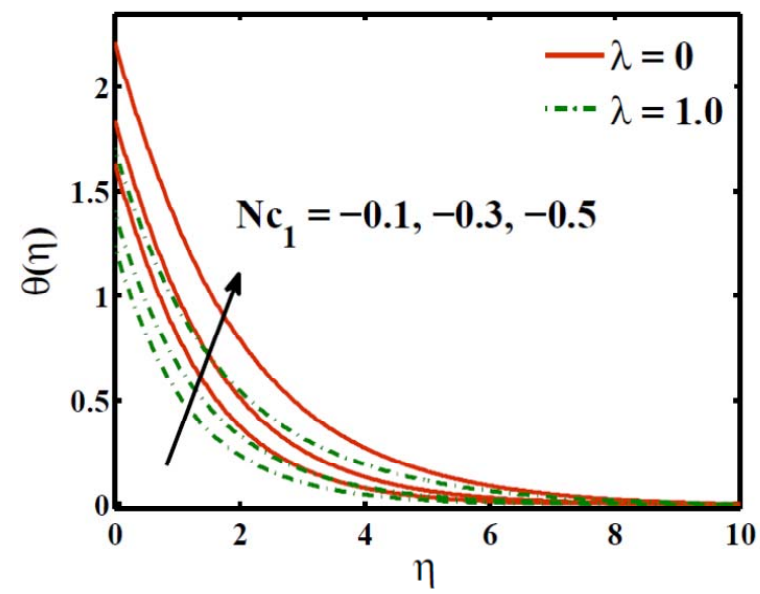

(b)Opposing flows

Fig.3. Effect of the buoyancy ratio of salt 1 and on the dimensionless temperature when $\operatorname{Ld}_{1}=1 ; \operatorname{Ld}_{2}=2$; $\mathrm{Le}_{1}=1 ; \mathrm{Le}_{2}=2 ; \mathrm{Ln}=2 ; \mathrm{Nb}=\mathrm{Nt}=\mathrm{Nr}=0.1 ; \delta=\pi / 6 ; \mathrm{Nd}_{1}=0.1 ; \mathrm{Nd}_{2}=0.2 ; \mathrm{Nc}_{2}=0.1$;

Figures 4 and 5 show the effects of Dufour-solutal Lewis numbers and buoyancy ratio of both salts on their respective dimensionless concentrations in the case of assisting flows. Within the boundary layer for larger Lewis numbers, the dimensionless concentrations of salts $1 \& 2$ are found to be higher. Figures $6 a$ and $6 \mathrm{~b}$ show the effect of the buoyancy ratio and nanofluid parameters on the nanoparticle volume fraction profile for the assisting flow. We note that increasing the Brownian motion parameter leads to a clustering of the nanoparticles near the plate. An increase in the Brownian motion of the nanoparticles leads to a decrease in the mass volume fraction profile of nanoparticles. The fast flow from the plate carries with it nanoparticles leading to an increase in the dimensionless concentration of nanoparticles. 


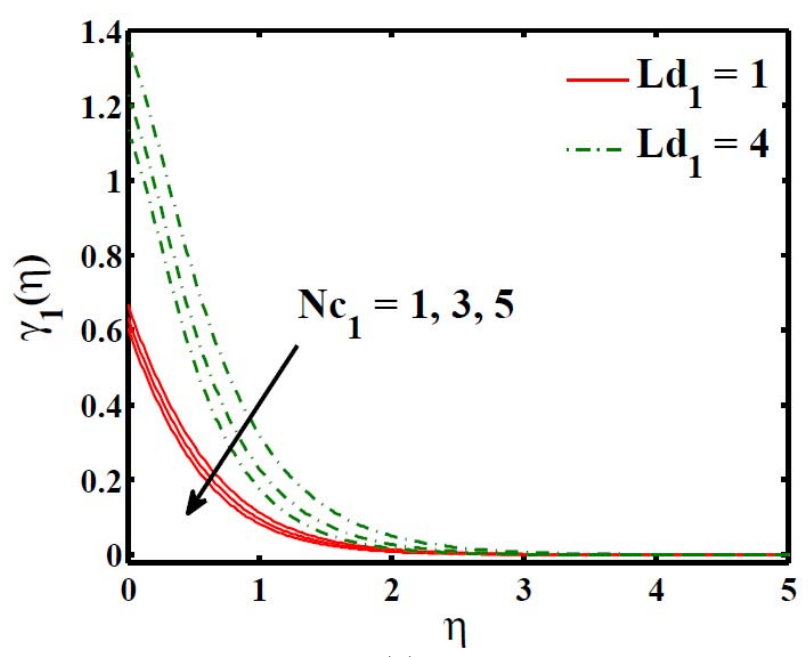

(a)

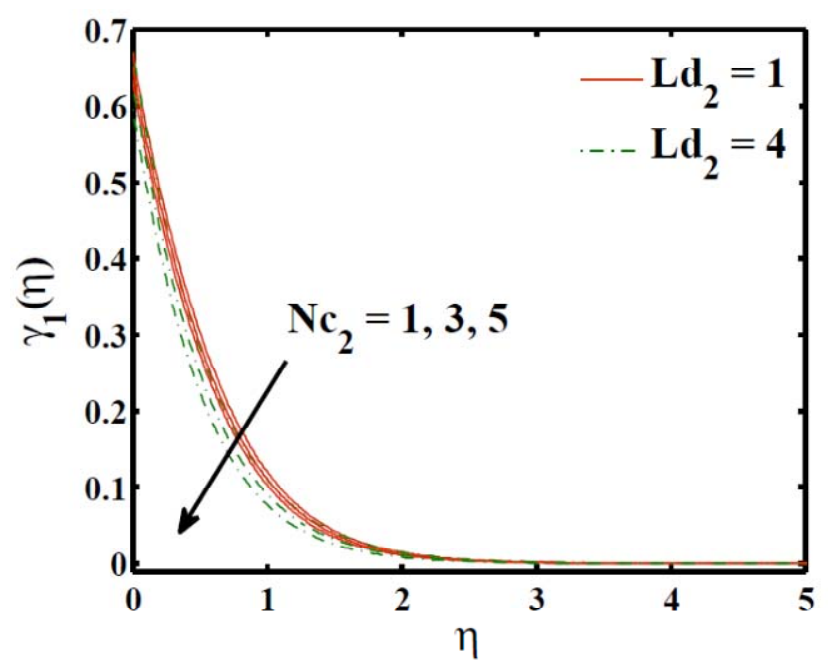

(b)

Fig.4. Effects of buoyancy ratios and Dufour-solutal Lewis numbers on the dimensionless concentration of salt 1 for the assisting flow when $\mathrm{Le}_{1}=1 ; \mathrm{Le}_{2}=2 ; \mathrm{Nd}_{1}=0.1 ; \mathrm{Nd}_{2}=0.2 ; \mathrm{Nb}=\mathrm{Nt}=\mathrm{Nr}=0.1 ; \mathrm{Ln}=2$; $\lambda=0.5 ; \delta=\pi / 6$.

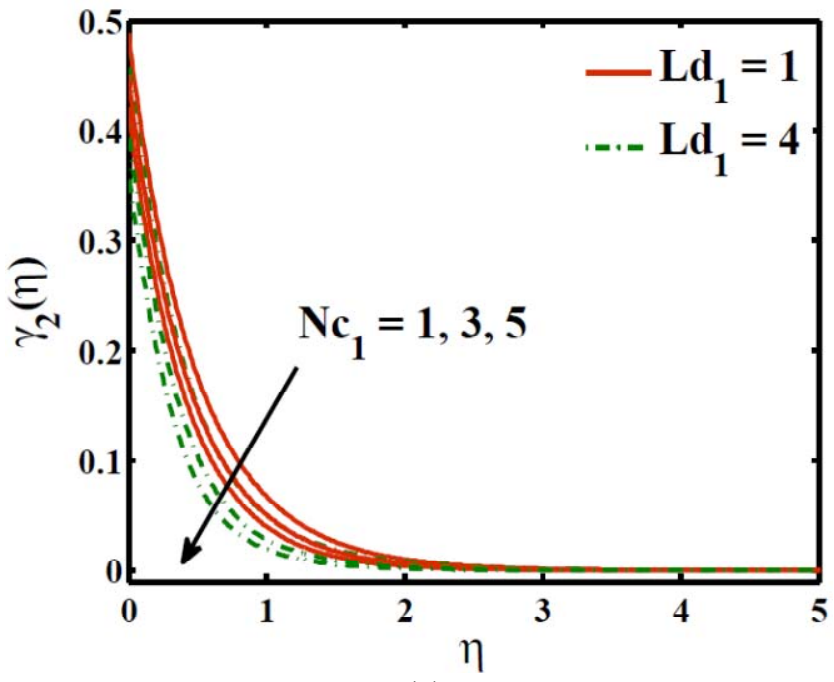

(a)

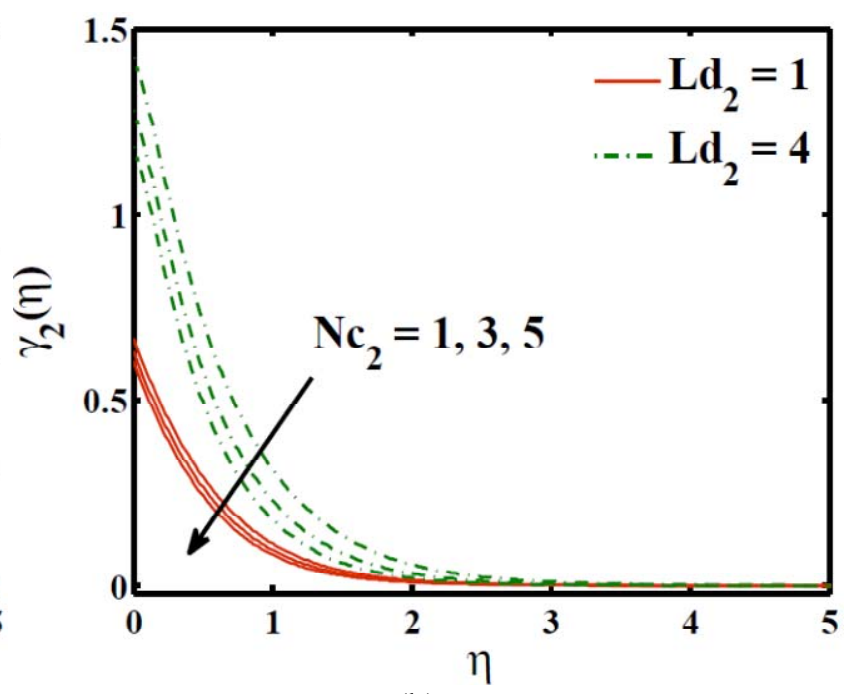

(b)

Fig.5. Effects of buoyancy ratios and Dufour-solutal Lewis numbers on the dimensionless concentration of salt 1 for the opposing flow when $\mathrm{Le}_{1}=1 ; \mathrm{Le}_{2}=2 ; \mathrm{Nd}_{1}=0.1 ; \mathrm{Nd}_{2}=0.2 ; \mathrm{Nb}=\mathrm{Nt}=\mathrm{Nr}=0.1 ; \mathrm{Ln}=2$; $\lambda=0.5 ; \delta=\pi / 6$. 


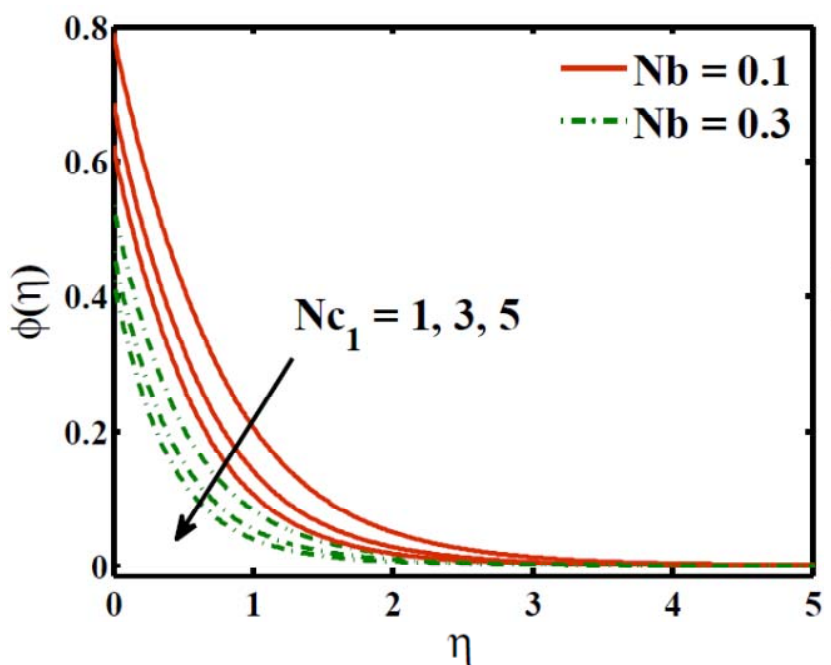

(a)

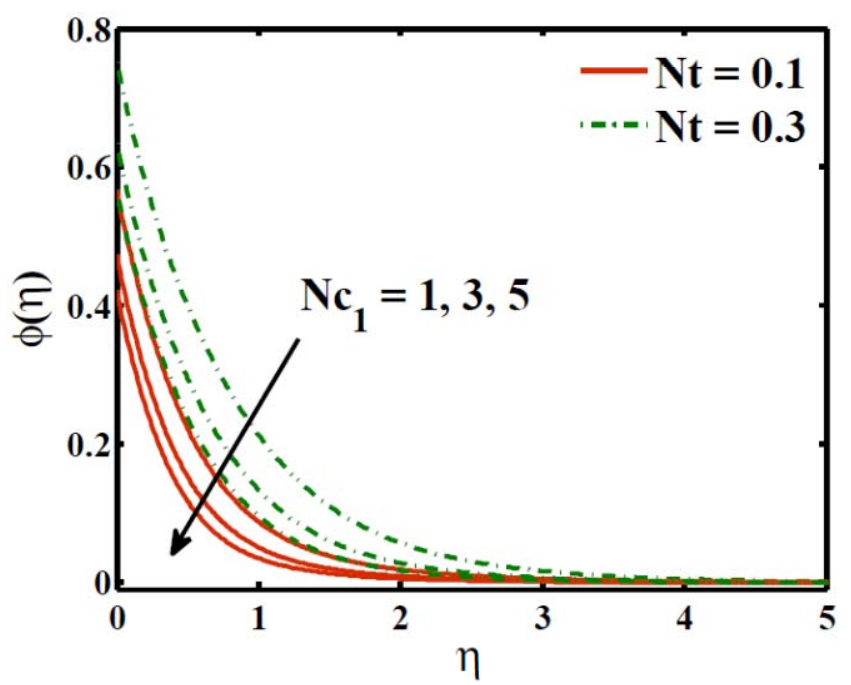

(b)

Fig.6. Effects of buoyancy ratios and nanofluid parameters on the dimensionless concentration of the nanofluid for the assisting flow when $\mathrm{Le}_{1}=1 ; \mathrm{Le}_{2}=2 ; \mathrm{Nd}_{1}=0.1 ; \mathrm{Nd}_{2}=0.2 ; \mathrm{Nb}=\mathrm{Nt}=\mathrm{Nr}=0.1$; $\operatorname{Ln}=2 ; \lambda=0.5 ; \delta=\pi / 6$.

The influence of angles of inclination from the vertical, $\delta$, ranging from 0 to $\frac{\pi}{2}$ on the temperature, solutal concentration of both salts and nanoparticle volume fraction are shown in Figs 7 and 8 . An increase in the angle of inclination leads to enhanced temperature, concentration of both salts, and nanoparticle volume fraction distributions within the boundary layer region. This is due to the reduction in the buoyancy effect caused by an increases in $\eta$.

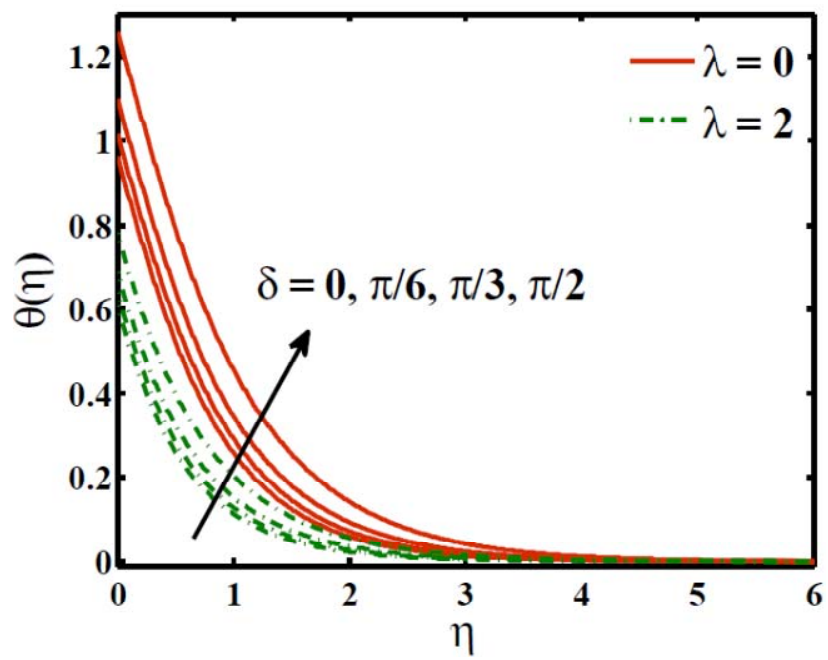

(a)

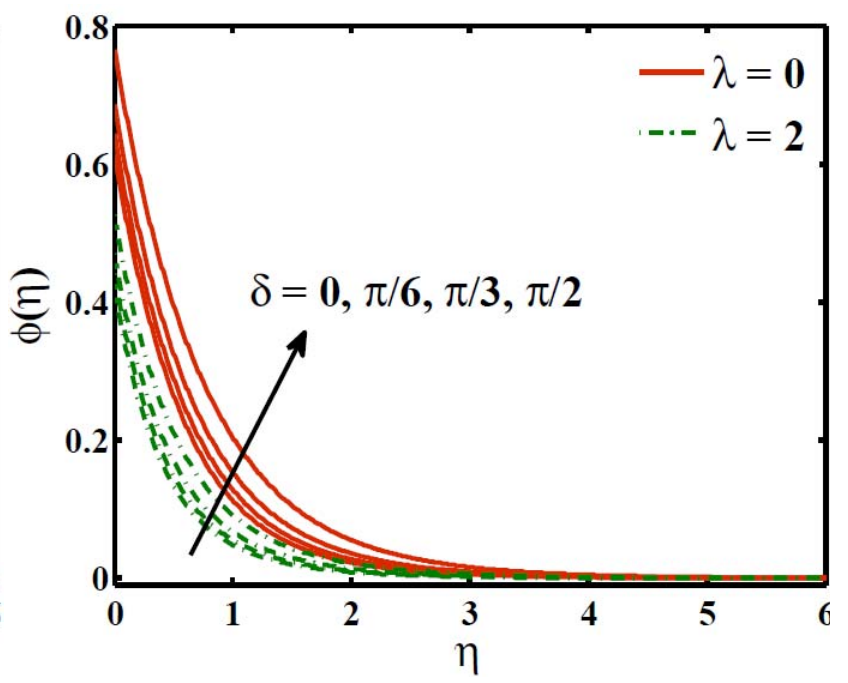

(b)

Fig.7. Effects of the angle of inclination $\delta$ and $\lambda$ on the dimensionless temperature and concentration of nanoparticles for the assisting flow when $\mathrm{Ld}_{1}=1 ; \mathrm{Ld}_{2}=2 ; \mathrm{Le}_{1}=1 ; \mathrm{Le}_{2}=2 ; \mathrm{Ln}=2 ; \mathrm{Nd}_{1}=0.1 ; \mathrm{Nd}_{2}=$ $0.2 ; \mathrm{Nc}_{1}=1 ; \mathrm{Nc}_{2}=2 ; \mathrm{Nr}=0.1 ; \mathrm{Nb}=0.3 ; \mathrm{Nt}=0.2$. 


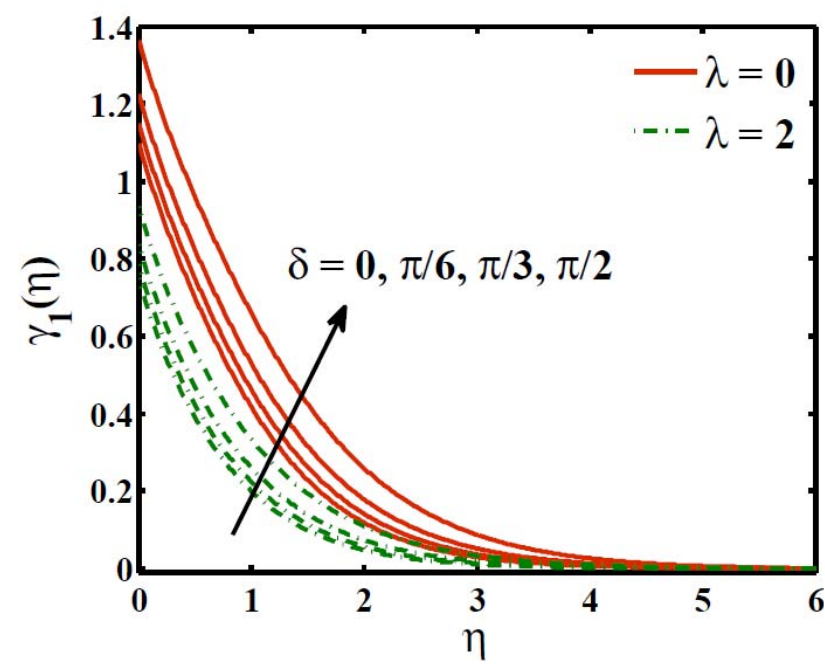

(a)

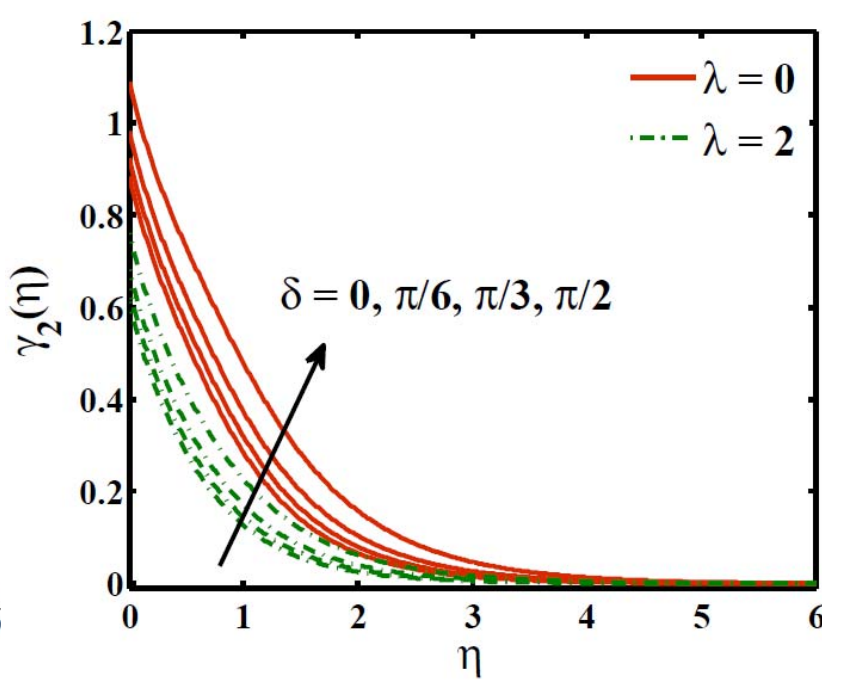

(b)

Fig.8. Effects of the angle of inclination $\delta$ and $\lambda$ on the dimensionless temperature and concentration of nanoparticles for the opposing flow when $\mathrm{Ld}_{1}=1 ; \mathrm{Ld}_{2}=2 ; \mathrm{Le}_{1}=1 ; \mathrm{Le}_{2}=2 ; \mathrm{Ln}=2 ; \mathrm{Nd}_{1}=0.1 ; \mathrm{Nd}_{2}$ $=0.2 ; \mathrm{Nc}_{1}=1 ; \mathrm{Nc}_{2}=2 ; \mathrm{Nr}=0.1 ; \mathrm{Nb}=0.3 ; \mathrm{Nt}=0.2$.

Figures $9 \mathrm{a}$ and $9 \mathrm{~b}$ show the effects of the Brownian motion parameter $\mathrm{Nb}$ on the reduced Nusselt number Nur and the reduced nanofluid Sherwood number as the nanofluid Lewis number Ln increases. Figures $10 \mathrm{a}$ and $10 \mathrm{~b}$ depict the effect of the thermophoretic parameter $\mathrm{Nt}$ on the heat and nanoparticles mass transfer rates for a variation of the nanofluid Lewis number Ln. The extension of this problem has also been done with the Hybrid approach (FEM + symbolic computation) by the authors.

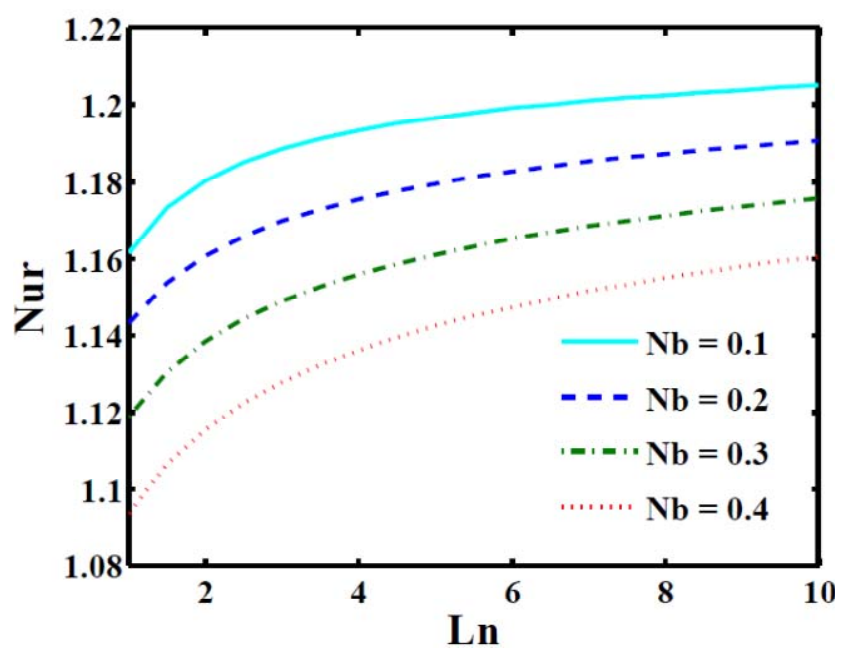

(a)

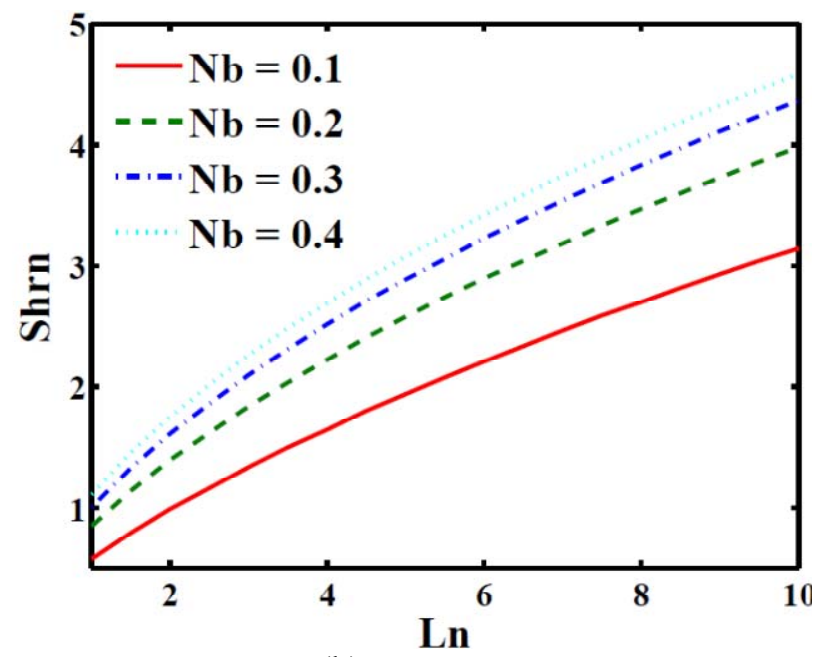

(b)

Fig.9. Effect of the nanofluid Lewis number $\mathrm{Ln}$ and the Brownian motion parameter $\mathrm{Nb}$ on the heat and nanoparticle mass transfer rates for assisting flow when $\mathrm{Ld}_{1}=1 ; \mathrm{Ld}_{2}=2 ; \mathrm{Le}_{1}=1 ; \mathrm{Le}_{2}=2 ; \mathrm{Nd}_{1}=0.1$; $\mathrm{Nd}_{2}=0.2 ; \mathrm{Nc}_{1}=1 ; \mathrm{Nc}_{2}=2 ; \mathrm{Nr}=0.1 ; \mathrm{Nt}=0.3 ; \lambda=0.5 ; \delta=\pi / 6$ 


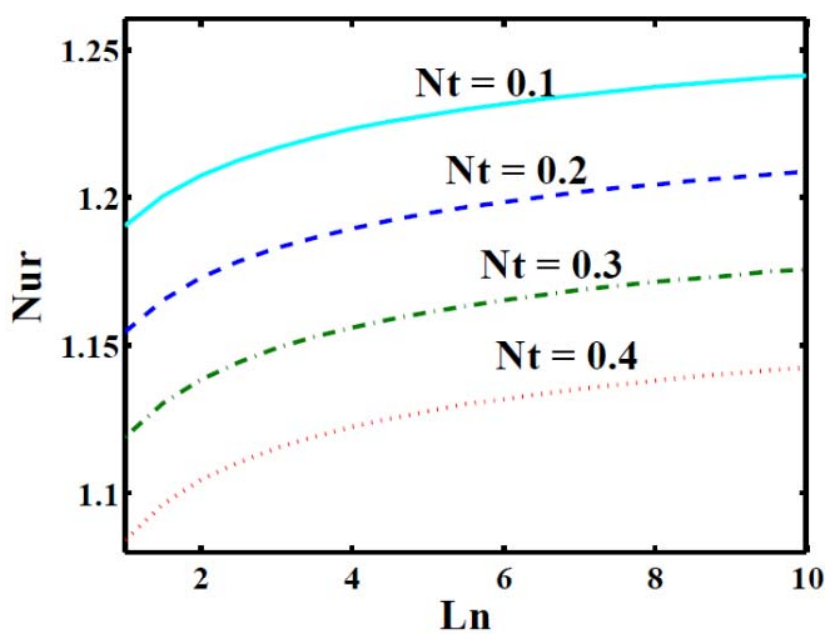

(a)

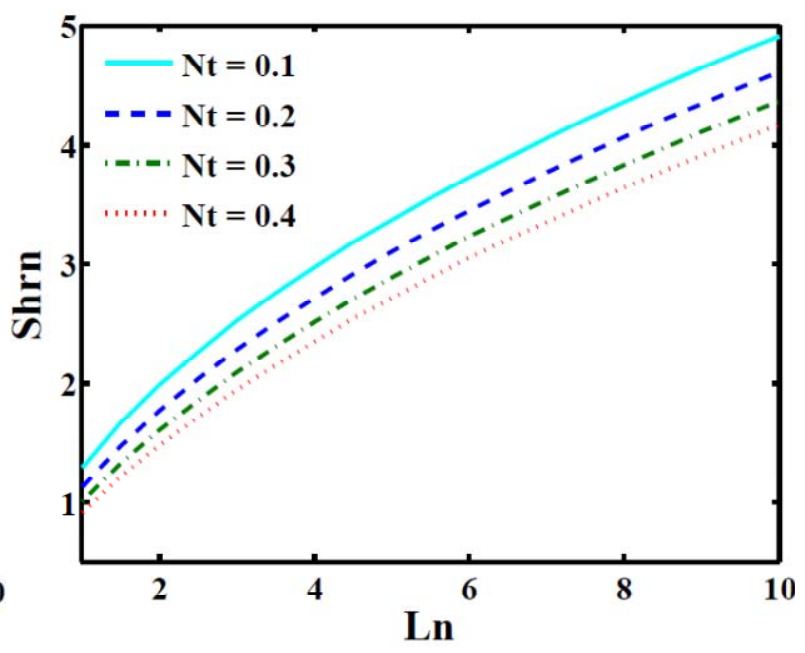

(b)

Fig.10. Effect of the nanofluid Lewis number Ln and the Thermophoresis parameter $\mathrm{Nt}$ on the heat and nanoparticle mass transfer rates for assisting flow when $\operatorname{Ld}_{1}=1 ; \operatorname{Ld}_{2}=2 ; \operatorname{Le}_{1}=1 ; \operatorname{Le}_{2}=2$; $\mathrm{Nd}_{1}=0.1 ; \mathrm{Nd}_{2}=0.2 ; \mathrm{Nc}_{1}=1 ; \mathrm{Nc}_{2}=2 ; \mathrm{Nr}=0.1 ; \mathrm{Nt}=0.3 ; \lambda=0.5 ; \delta=\pi / 6$

\title{
6. Conclusion
}

The result can be summarized as follows:

- The addition of nanoparticles and salts in the base fluid increases the heat transfer rates. Thus, useful in fast cooling on the drug delivery with induction of nanoparticles.

- Temperature decreases with the buoyancy ratio of salt 1 for assisting flows but an opposite trend is found in the case of opposing flows.

- Increasing the thermophoresis parameter (Nt) increases nanoparticle concentration, whereas increasing the Brownian motion parameter $(\mathrm{Nb})$ decreases the concentration of nanoparticles. So, nano mass transfer rates increase with $\mathrm{Nb}$ but decrease with $\mathrm{Nt}$.

- An increase in the angle of inclination of the inclined surface reduces the heat, regular mass, and nanoparticle mass transfer rates between the porous medium and the inclined surface. This is an important conclusion, for efficient drug delivery where the direction of incision has an important role.

- The effect of variation of the Brownian motion or the thermophoresis parameter on the heat transfer rate is larger than that of the buoyancy ratio parameter, implying the effectiveness of nanofluids.

\section{Acknowledgement}

The second author (R. Goyal) would like to thank DST for providing PhD scholarship.

\section{Nomenclature}

\author{
$C$ - concentration \\ $C_{p}$ - specific heat at constant pressure \\ $D_{S 1}, D_{S 2} \quad$ - solutal diffusivity \\ $\mathrm{D}_{\mathrm{B}} \quad$ - Brownian motion coefficient \\ $D_{T} \quad$ - thermophoretic coefficient \\ $\mathrm{D}_{\mathrm{TC}} \quad$ - Dufour type diffusivity
}




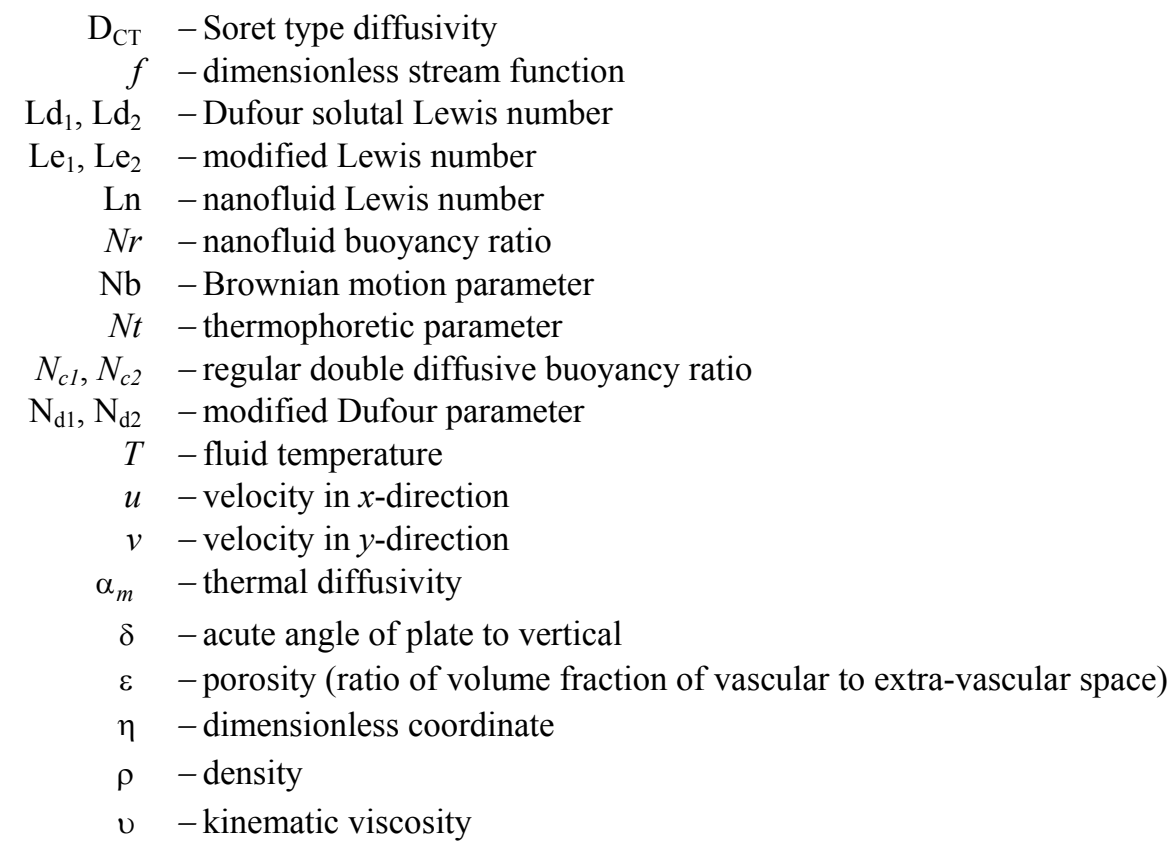

Subscripts

$f$ - base fluid

nf - nanofluid

\section{References}

[1] Nield D. and Bejan A. (2006): Convection in Porous Media. - third ed., New York: Springer.

[2] Pop I. and Ingham D. (2001): Convective Heat Transfer: Mathematical and Computational Modeling of Viscous Fluids and Porous Media. - Oxford: Pergamon.

[3] Ingham D. and Pop I. (2005): Transport Phenomena in Porous Media III. - Oxford: Elsevier.

[4] Choi S.U.S. and Eastman J.A. (1995): Enhancing thermal conductivity of fluids with nanoparticles in developments and applications of non-newtonian flows. - ASME FED, vol.231/MD-vol.66, pp.99-105.

[5] Choi S.U.S., Zhang Z.G., Yu W., Lockwood F.E. and Grulke E.A. (2001): Anomalous thermal conductivity enhancement in nanotube suspensions. - Applied Physics Letters, vol.79, No.14, pp.2252-2254.

[6] Masuda H., Ebata A., Teramae K. and Hishinuma N. (1993): Alteration of thermal conductivity and viscosity of liquid by dispersing ultra-nano particles. - Netsu Bussei, vol.7, No.4, pp.227-233.

[7] Buongiorno J. (2006): Convective transport in nanofluids. - Journal of Heat Transfer, vol.128, No.3, pp.240250.

[8] Kuznetsov A. and Nield D. (2010): Natural convective boundary-layer flow of a nanofluid past a vertical plate. - International Journal of Thermal Sciences, vol.49, pp.243-247.

[9] Nield D. and Kuznetsov A. (2009): The Cheng-Minkowycz problem for natural convective boundary-layer flow in a porous medium saturated by a nanofluid. - International Journal of Heat and Mass Transfer, vol.52, pp.5792-5795.

[10] Cheng P. and Minkowycz W. (1977): Free convection about a vertical plate embedded in a porous medium with application to heat transfer from a dike. - Journal of Geophysical Research, vol.82, pp.2040-2044. 
[11] Khan W. and Pop I. (2010): Boundary-layer flow of a nanofluid past a stretching sheet. - International Journal of Heat and Mass Transfer, vol.53, pp.2477-2483.

[12] Khan W. and Aziz A. (2011): Double-diffusive natural convective boundary layer flow in a porous medium saturated with a nanofluid over a vertical plate: Prescribed surface heat, solute and nanoparticle fluxes, International Journal of Thermal Sciences, vol.50, pp.2154-2160.

[13] Kuznetsov A. and Nield D. (2011): Double-diffusive natural convective boundary-layer flow of a nanofluid past a vertical plate. - International Journal of Thermal Sciences, vol.50, No.5, pp.712-717.

[14] Rana P., Bhargava R. and Beg O.A. (2012): Numerical solution for mixed convection boundary layer flow of a nanofluid along an inclined plate embedded in a porous medium. - Computers and Mathematics with Applications, vol.64, pp.2816-2832.

[15] Murthy P.V.S.N., Sutradhar A. and RamReddy C. (2013): Double-diffusive free convection flow past an inclined plate embedded in a non-Darcy porous medium saturated with a nanofluid. - Transport in Porous Medium, vol.98, pp.553-564.

[16] Khan Z., Khan W. and Pop I. (2013): Triple diffusive free convection along a horizontal plate in porous media saturated by a nanofluid with convective boundary condition. - International Journal of Heat and Mass Transfer, vol.66, pp.603-612.

[17] Nield D. and Kuznetsov A. (2011): The Cheng-Minkowycz problem for the double-diffusive natural convective boundary-layer flow in a porous medium saturated with a nanofluid. - International Journal of Heat and Mass Transfer, vol.54, pp.374-378.

[18] Wu M., Kuznetsov A.K. and Jasper W. (2010): Modelling of particle trajectories in an electrostatically charged channel. - Physics of Fluids, vol.22, 043301.

[19] Wu G., Kuznetsov A.K. and Jasper W. (2011): Distribution characteristics of exhaust gases and soot particles in a wall-flow ceramics filter. - Journal of Aerosol Science, vol.42, pp.447-461.

[20] Rionero S. (2013): Triple diffusive convection in porous media. - Acta Mechanica, vol.224, No.2, pp.447-458. URL http://dx.doi.org/10.1007/s00707-012-0749-2.

[21] Reddy J.N. (1985): An Introduction to the Finite Element Method. - New York: McGraw-Hill Book Co.

[22] Lin Y.Y. and Lo S.P. (2003): Finite element modeling for chemical mechanical polishing process under different back pressures. - Journal of Materials Processing Technology, vol.140, No.1-3, pp.646-652.

[23] Dettmer W. and Peric D. (2006): A computational framework for fluid-rigid body interaction: Finite element formulation and applications. - Computer Methods in Applied Mechanics and Engineering, vol.195, No.1316, pp.1633-1666.

[24] Hansbo A. and Hansbo P. (2004): A finite element method for the simulation of strong and weak discontinuities in solid mechanics. - Computer Methods in Applied Mechanics and Engineering, vol.193, No.33-35, pp.35233540 .

[25] Noghrehabadi A., Behseresht A. and Ghalambaz M. (2013): Natural convection of nanofluid over vertical plate embedded in porous medium: prescribed surface heat flux. - Applied Mathematics and Mechanics, vol.34, pp.669-686.

Received: April 22, 2016

Revised: June 27, 2017 